\title{
Evaluating the numbers of some skew standard Young tableaux of truncated shapes
}

\author{
Ping Sun* \\ Submitted: Nov 19, 2013; Accepted: Dec 6, 2014; Published: Jan 2, 2015 \\ Mathematics Subject Classifications: 05D40, 05A15, 05E15
}

\begin{abstract}
In this paper the number of standard Young tableaux (SYT) is evaluated by the methods of multiple integrals and combinatorial summations. We obtain the product formulas of the numbers of skew SYT of certain truncated shapes, including the skew SYT $\left((n+k)^{r+1}, n^{m-1}\right) /(n-1)^{r}$ truncated by a rectangle or nearly a rectangle, the skew SYT of truncated shape $\left((n+1)^{3}, n^{m-2}\right) /(n-2) \backslash\left(2^{2}\right)$, and the SYT of truncated shape $\left((n+1)^{2}, n^{m-2}\right) \backslash(2)$.
\end{abstract}

Keywords: truncated shapes; standard Young tableaux; order statistics; Selberg integral

\section{Introduction}

The enumeration of standard Young tableaux (SYT) is a fundamental problem in enumerative combinatorics. The number of SYT is given by the well-known hook-length formulas [5]. However hook-length formulas cannot give the number of SYTs of truncated shapes. Two recent papers $[1,8]$ counted the number of SYT of certain truncated shapes, including a rectangle truncated by a shifted staircase and a rectangle truncated by a square minus a box. The corresponding product formulas are derived by different combinatorial methods: Adin et al's pivoting theory in [1] and Panova's bijective arguments with Schur functions identities in [8].

Recall that a partition $\lambda$ of a positive integer $n$ is a non-increasing sequence of nonnegative integers $\lambda=\left(\lambda_{1}, \cdots, \lambda_{d}\right)$ such that $n=\lambda_{1}+\cdots+\lambda_{d}$. A Ferrers diagram of shape $\lambda$ is a left-justified array of $n$ boxes, with row $i$ (from top to bottom) containing $\lambda_{i}$ boxes. $\lambda$ is used to represent both the partition and the shape, while $(i, j)$ denotes the box in row $i$ and column $j$.

Let $\lambda=\left(\lambda_{1}, \lambda_{2}, \cdots\right), \mu=\left(\mu_{1}, \mu_{2}, \cdots\right)$ and $\nu=\left(\nu_{1}, \nu_{2}, \cdots\right)$ be integer partitions such that $\lambda_{i}>\mu_{i}, \lambda_{i}>\nu_{i}$. A skew shape $\lambda / \mu$ is an array of boxes where $\mu_{i}$ boxes are

\footnotetext{
*Department of Mathematics, Northeastern University, Shenyang, China; plsun@mail.neu.edu.cn
} 
deleted from the beginning of row $i$. A standard Young tableau of shape $\lambda$ is a labeling by $\{1,2, \cdots,|\lambda|\}$ of the boxes in the Ferrers diagram such that each row and column is increasing (from left to right and from top to bottom respectively). The standard shifted Young tableau of shape $\lambda$ is like the ordinary shape except that row $i$ starts with its leftmost box in position $(i, i)$ if $\lambda_{1}>\lambda_{2}>\cdots>\lambda_{d}$. The number of SYT of shape $\lambda$ is denoted by $f^{\lambda}$. (More properties of SYT can be found in [12])

A diagram of truncated shape $\lambda \backslash \nu$ is a left-justified array of boxes where $\nu_{i}$ boxes are deleted from the end of row $i$. A SYT of truncated shape $\lambda \backslash \nu$ is a filling of the corresponding truncated diagram with the integers from 1 to $|\lambda|-|\nu|$ such that each row and column is increasing. Generally, a SYT-type chart of shape $\lambda$ is a labeling by $\{1,2, \cdots,|\lambda|\}$ in a collection of boxes which may not be left-justified or connected, preserving the property that each row and column is increasing. SYT, skew SYT and SYT of truncated shape are SYT-type charts. For example, the SYT-type chart of shape $\left(4^{2}, 3\right) /\left(1^{2}\right) \backslash(1,3) \cup(2,2)$, which is the skew SYT of shape $\left(4^{2}, 3\right) /\left(1^{2}\right)$ with $(1,3)$ and $(2,2)$ removed, is illustrated as

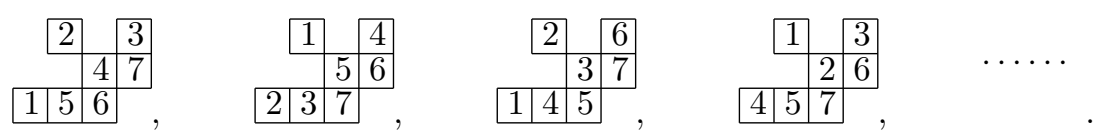

This paper is organized as follows. In Section 2 we introduce an order statistics model of SYT-type chart and present several multiple integral formulas applying to the enumeration of SYT-type chart. In Section 3 we give some enumerating results implied directly by Selberg integral, including two results of Adin et al., Panova, and the numbers of skew SYT of shape $\left.(n+k)^{r+1}, n^{m-1}\right) /(n-1)^{r}$ truncated by a rectangle or nearly a rectangle. In Section 4 we derive several combinatorial identities, which are used to the enumerations of the skew SYT of truncated shape $\left((n+1)^{3}, n^{m-2}\right) /(n-2) \backslash\left(2^{2}\right)$ and the SYT of truncated shape $\left((n+1)^{2}, n^{m-2}\right) \backslash(2)$. All the corresponding product formulas are given.

\section{Order statistics, multiple integrals and enumeration of SYT- type charts}

It is known that integration methods for enumeration have been used by A. Regev [9], R. P. Stanley [11] and others [4, 2], which may be called "order polytope" model because the number of SYT of a given shape (or alternating permutation) is interpreted as a volume of a corresponding order polytope. From the point of view of statistics, each row of a SYT-type chart corresponds to a group of independent order statistics. Then the number of SYT-type chart will also be interpreted as distribution of nested order statistics in this paper.

Let $\xi_{1}, \cdots, \xi_{n}$ be $n$ independent and identically distributed random variables with uniform distribution on interval $(0,1)$, the corresponding order statistics are obtained by 
arranging these $n \xi$ 's in nondecreasing order, and are denoted by $\left(\xi_{1, n}, \cdots, \xi_{n, n}\right)$ such that $\xi_{1, n}<\cdots<\xi_{n, n}$ (For details, see [3]). Suppose that there are $d$ groups of independent order statistics $\left(\xi_{1, \lambda_{j}}^{(j)}, \cdots, \xi_{\lambda_{j}, \lambda_{j}}^{(j)}\right)(1 \leqslant j \leqslant d)$ from uniform distribution on $(0,1)$, they will be nested if there be $r_{j}>j$ such that $\xi_{1, \lambda_{j}}^{(j)}<\xi_{k_{j}, \lambda_{r_{j}}}^{\left(r_{r}\right)}$ for $1 \leqslant j \leqslant d-1$.

Consider the distribution of nest order statistics

$$
\mathbf{P}_{\lambda}=\mathbf{P}\left\{\bigcap_{j=1}^{d-1}\left(\xi_{1, \lambda_{j}}^{(j)}<\xi_{k_{j}, \lambda_{r_{j}}}^{\left(r_{j}\right)}, \xi_{2, \lambda_{j}}^{(j)}<\cdots, \xi_{\lambda_{j}, \lambda_{j}}^{(j)}<c_{j}\right)\right\}
$$

where $c_{j}$ may be $\xi_{i_{j}, \lambda_{s_{j}}}^{\left(s_{j}\right)}\left(s_{j} \geqslant r_{j}\right)$ or 1 , illustrated as the following

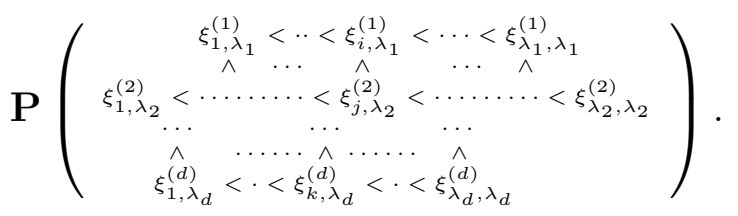

Let $S_{\lambda}$ denote the corresponding nested simplex of nested order statistics:

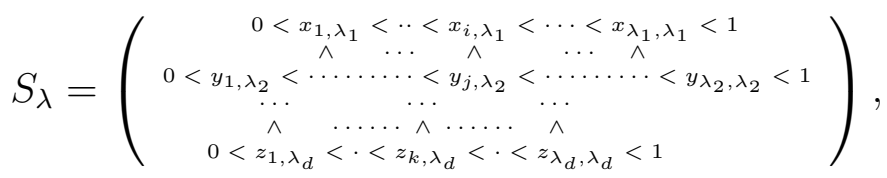

it follows from the joint probability density function of $\left(\xi_{1, \lambda_{1}}^{(1)}, \cdots, \xi_{\lambda_{d}, \lambda_{d}}^{(d)}\right)[3]$ that

$$
\mathbf{P}_{\lambda}=\prod_{i=1}^{d} \lambda_{i} ! \iint_{S_{\lambda}} \cdots \int d x_{1, \lambda_{1}} \cdots d z_{\lambda_{d}, \lambda_{d}}
$$

On the other hand, for each sample point $\omega$, the event

$$
A=\left\{\omega: \bigcap_{j=1}^{d-1}\left(\xi_{1, \lambda_{j}}^{(j)}(\omega)<\xi_{k_{j}, \lambda_{r_{j}}}^{\left(r_{j}\right)}(\omega), \xi_{2, \lambda_{j}}^{(j)}(\omega)<\cdots, \xi_{\lambda_{j}, \lambda_{j}}^{(j)}(\omega)<c_{j}(\omega)\right)\right\}
$$

corresponds to a filling of the SYT-type chart of shape $\lambda$, the property of independent and identically distributed makes sure that all the outcomes of event $A$ are equally likely. Based on this discrete structure, there is

$$
\mathbf{P}_{\lambda}=\frac{N_{\lambda}}{\left(\begin{array}{c}
|\lambda| \\
\lambda_{1}, \lambda_{2}, \cdots, \lambda_{d}
\end{array}\right)}
$$

where $N_{\lambda}$ is the number of SYT-type chart of shape $\lambda$.

Therefore, $N_{\lambda}$ could be evaluated by

$$
N_{\lambda}=|\lambda| ! \operatorname{Vol}\left(S_{\lambda}\right),
$$


where $\operatorname{Vol}\left(S_{\lambda}\right)$ is the volume of the corresponding nested simplex of SYT-type chart of shape $\lambda$.

The formula (1) shows that not only the number of regular SYT, but also the number of SYT of truncated shape and other variations can be obtained by evaluating some specific multiple integrals. For example, Consider three groups of independent order statistics $\left(\xi_{1,2}, \xi_{2,2}\right),\left(\eta_{1,2}, \eta_{2,2}\right),\left(\tau_{1,3}, \tau_{2,3}, \tau_{3,3}\right)$ from uniform distribution on $(0,1)$, the number of the SYT-type chart of shape $\left(4^{2}, 3\right) /\left(1^{2}\right) \backslash(1,3) \cup(2,2)$ is given by

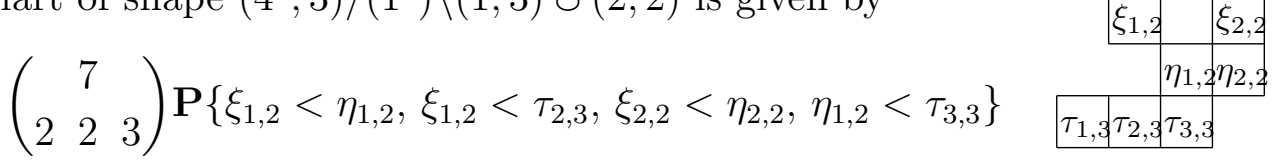

$$
\begin{aligned}
& =7 ! \quad \int \cdots \int_{\substack{x_{1,2}<x_{2,3}, x_{1,2}<x_{3,2}, x_{2,3}<x_{3,3}, x_{1,4}<x_{2,4} \\
0<x_{1,2}<x_{1,4}<1,0<x_{2,3}<x_{2,4}<1,0<x_{3,1}<x_{3,2}<x_{3,3}<1}} d x_{1,1} \cdots d x_{3,3}=51,
\end{aligned}
$$

where $x_{i, j}$ is the corresponding variable of the box $(i, j)$ in the SYT-type chart of shape $\left(4^{2}, 3\right) /\left(1^{2}\right) \backslash(1,3) \cup(2,2)$.

This paper considers the enumeration of skew SYT of truncated shapes by using of (1). For convenient, we use the following notation as in $[1,8]$ :

$$
F_{n}:=\prod_{i=1}^{n-1} i !, \quad F_{0}=1
$$

If necessary, $\operatorname{det}\left(a_{i j}\right)_{i, j=1}^{m}$ may be written to be $\operatorname{det}\left(\begin{array}{c}a_{i j}, 1 \leqslant i \leqslant k \\ a_{i j}, k+1 \leqslant i \leqslant m\end{array}\right)_{j=1}^{m}$.

The following Lemma 1 presents two multiple integrals which are evaluated by integrating over the lower left corner of a rectangular shape, illustrated as
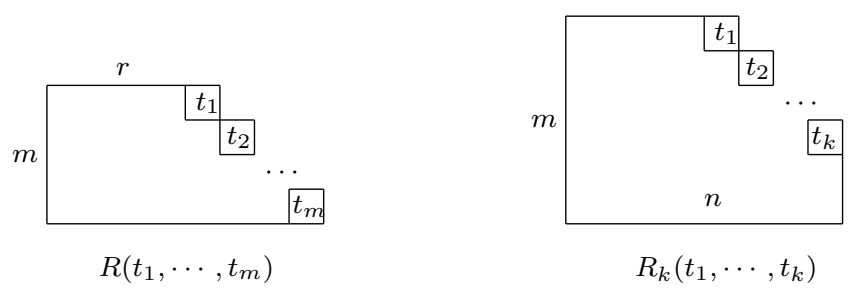

Lemma 1. For $m \geqslant 2, r \geqslant 0,0<t_{1} \leqslant \cdots \leqslant t_{m} \leqslant 1$, we have the following multiple integral formula

$$
\begin{aligned}
& R\left(t_{1}, \cdots, t_{m}\right)=\underset{0<x_{i, 1}<\cdots<x_{i, r+i-1}<t_{i}, 1 \leqslant i \leqslant m}{\int} \cdots \int_{1 \leqslant j} d x_{1,1} \cdots d x_{m, r+m-1} \\
& \begin{array}{c}
x_{1, j}<\cdots<x_{m, j}, \quad 1 \leqslant j \leqslant r \\
t_{j-r}<x_{j-r+1, j}<\cdots<x_{m, j}, \quad r+1 \leqslant j \leqslant r+m-1
\end{array} \\
& =\operatorname{det}\left(\frac{t_{i}^{r+j-1}}{(r+j-1) !}\right)_{i, j=1}^{m}=\frac{F_{r}}{F_{r+m}} \prod_{i=1}^{m} t_{i}^{r} \cdot \prod_{1 \leqslant i<j \leqslant m}\left(t_{j}-t_{i}\right) .
\end{aligned}
$$


Furthermore, for $n \geqslant m>k \geqslant 1$,

$$
\begin{aligned}
R_{k}\left(t_{1}, \cdots, t_{k}\right) & \int \underset{\substack{0<x_{i, 1}<\cdots<x_{i, n-k+i-1<t_{i}, 1 \leqslant i \leqslant k} \\
0<x_{i, 1}<\cdots<x_{i, n}<1, k+1 \leqslant i \leqslant m ; x_{1, j}<\cdots<x_{m, j}, 1 \leqslant j \leqslant n-k \\
t_{j-n+k}<x_{j-n+k+1, j}<\cdots<x_{m, j}, n-k+1 \leqslant j \leqslant n}}{m} d x_{1,1} \cdots d x_{m, n} \\
& =\operatorname{det}\left(\begin{array}{c}
\frac{t_{i}^{n-k+j-1}(n-k+j-1) !}{(n-k+j)}, 1 \leqslant i \leqslant k \\
\frac{1}{(n-i+j) !}, k+1 \leqslant i \leqslant m
\end{array}\right)_{j=1}^{m} \\
& =\frac{F_{n-k} F_{m-k}}{F_{n+m-k}} \prod_{i=1}^{k} t_{i}^{n-k}\left(1-t_{i}\right)^{m-k} \cdot \prod_{1 \leqslant i<j \leqslant k}\left(t_{j}-t_{i}\right) .
\end{aligned}
$$

Proof. We observe that

$$
\int_{x_{m-1,1}}^{x_{m, 2}} d x_{m, 1}=\left|\begin{array}{cc}
1 & x_{m-1,1} \\
1 & x_{m, 2}
\end{array}\right|=\left|\begin{array}{ccc}
1 & x_{m-2,1} & \frac{x_{m-2,1}^{2}}{2 !} \\
0 & 1 & x_{m-1,1} \\
0 & 1 & x_{m, 2}
\end{array}\right|
$$

then,

$$
\begin{gathered}
\iiint_{\substack{x_{m-1,1}<x_{m, 1}<x_{m, 2} \\
x_{m-2,1}<x_{m-1,1}<x_{m-1,2}<x_{m, 2}<x_{m, 3}}} d x_{m, 1} d x_{m-1,1} d x_{m, 2} \\
=\left|\begin{array}{ccc}
1 & x_{m-2,1} & \frac{x_{m-2,1}^{2}}{2 !} \\
1 & x_{m-1,2} & \frac{x_{m-1,2}^{2}}{2 !} \\
1 & x_{m, 3} & \frac{x_{m, 3}^{2}}{2 !}
\end{array}\right|=\left|\begin{array}{cccc}
1 & x_{m-3,1} & \frac{x_{m-3,1}^{2}}{2 !} & \frac{x_{m-3,1}^{3}}{3 !} \\
0 & 1 & x_{m-2,1} & \frac{x_{m-2,1}^{2}}{2 !} \\
0 & 1 & x_{m-1,2} & \frac{x_{m-1,2}^{2}}{2 !} \\
0 & 1 & x_{m, 3} & \frac{x_{m, 3}^{2}}{2 !}
\end{array}\right|,
\end{gathered}
$$

and we have

$$
\int_{\substack{x_{i-1, j}<x_{i, j}<x_{i, j+1} \\ 2 \leqslant i \leqslant m, 1 \leqslant j \leqslant m-1}} d x_{m, 1} \cdots d x_{2,1} d x_{3,2} \cdots d x_{m, m-1}=\operatorname{det}\left(\frac{x_{i, i}^{j-1}}{(j-1) !}\right)_{i, j=1}^{m}
$$

which gives the determinant representation for $R\left(t_{1}, \cdots, t_{m}\right)$ in case of $r=0$. Furthermore, integrating respect to $x_{1, j}, x_{2, j+1}, \cdots, x_{m, j+m-1}(j=1,2, \cdots, r)$ gives the determinant representation for $R\left(t_{1}, \cdots, t_{m}\right)$. Finally, (2) follows from Vandermonde determinant.

A similar argument shows that the determinant representation for $R_{k}\left(t_{1}, \cdots, t_{k}\right)$ holds, therefore we have

$$
R_{k}\left(t_{1}, \cdots, t_{k}\right)=\frac{F_{n-k}}{F_{n+m-k}} \prod_{i=1}^{k} t_{i}^{n-k} \cdot \operatorname{det}\left(\begin{array}{cc}
t_{i}^{j-1}, & 1 \leqslant i \leqslant k \\
(n-k+j-1)_{i-k-1}, & k+1 \leqslant i \leqslant m
\end{array}\right)_{j=1}^{m} .
$$


Noticing that $(x)_{r}:=x(x-1) \cdots(x-r+1)=\sum_{i=0}^{r} s(r, i) x^{i}$ and $(x)_{0}=1$, where $s(r, i)$ is Stirling numbers of the first kind. For any real number $x$,

$\operatorname{det}\left(\begin{array}{cc}a_{i j}, & 1 \leqslant i \leqslant k \\ (x+j)_{i-k-1}, & k+1 \leqslant i \leqslant m\end{array}\right)^{m}=\operatorname{det}\left(\begin{array}{cc}a_{i j}, & 1 \leqslant i \leqslant k \\ (x+j)^{i-k-1}, & k+1 \leqslant i \leqslant m\end{array}\right)^{m}$,

and the second determinant in above is easy to be simplified to triangular form:

$$
\operatorname{det}\left(\begin{array}{cc}
a_{i j}, & 1 \leqslant i \leqslant k \\
(j-1)_{i-k-1}, & k+1 \leqslant i \leqslant m
\end{array}\right)_{j=1}^{m} .
$$

To prove (3), it suffices to evaluate the following determinant

$$
D_{m}=\operatorname{det}\left(\begin{array}{cc}
t_{i}^{j-1}, & 1 \leqslant i \leqslant k \\
(j-1)_{i-k-1}, & k+1 \leqslant i \leqslant m
\end{array}\right)_{j=1}^{m},
$$

$D_{m}$ is simplified by the $i$-th row subtracting the $(k+1)$-th row for $1 \leqslant i \leqslant k$, and then the $(j+1)$-th column subtracting the $j$-th column for $2 \leqslant j \leqslant m-1$. Therefore,

$$
D_{m}=(m-k-1) ! \prod_{i=1}^{k}\left(1-t_{i}\right) \times D_{m-1}=\cdots=F_{m-k} \prod_{i=1}^{k}\left(1-t_{i}\right)^{m-k} \cdot \prod_{1 \leqslant i<j \leqslant k}\left(t_{j}-t_{i}\right) .
$$

The proof of lemma 1 is complete.

Furthermore, Selberg's integral which is a generalization of Euler's beta integral

$$
\int_{0}^{1} x^{\alpha-1}(1-x)^{\beta-1} d x=\frac{\Gamma(\alpha) \Gamma(\beta)}{\Gamma(\alpha+\beta)}, \quad \alpha>0, \beta>0
$$

is shown to be useful in this paper.

Lemma 2. [10, 6] For $\operatorname{Re}(\alpha)>0, \operatorname{Re}(\beta)>0, \operatorname{Re}(\gamma)>-\min \left\{\frac{1}{k}, \frac{\operatorname{Re}(\alpha)}{k-1}, \frac{\operatorname{Re}(\beta)}{k-1}\right\}$,

$$
\begin{aligned}
S_{k}(\alpha, \beta, \gamma) & =\int_{0}^{1} \cdots \int_{0}^{1} \prod_{i=1}^{k} t_{i}^{\alpha-1}\left(1-t_{i}\right)^{\beta-1} \prod_{1 \leqslant i<j \leqslant k}\left|t_{i}-t_{j}\right|^{2 \gamma} d t_{1} \cdots d t_{k} \\
& =k ! \prod_{j=0}^{k-1} \frac{\Gamma(\alpha+j \gamma) \Gamma(\beta+j \gamma) \Gamma(\gamma+j \gamma)}{\Gamma(\alpha+\beta+(k+j-1) \gamma) \Gamma(\gamma)}
\end{aligned}
$$

Specially, the equivalent form of Selberg integral used in this paper is

$$
\begin{aligned}
& \int_{0<t_{1}<\cdots<t_{k}<1} \cdots \prod_{i=1}^{k} t_{i}^{\alpha-1}\left(1-t_{i}\right)^{\beta-1} \prod_{1 \leqslant i<j \leqslant k}\left(t_{j}-t_{i}\right)^{2 \gamma} d t_{1} \cdots d t_{k} \\
= & \prod_{j=0}^{k-1} \frac{\Gamma(\alpha+j \gamma) \Gamma(\beta+j \gamma) \Gamma(\gamma+j \gamma)}{\Gamma(\alpha+\beta+(k+j-1) \gamma) \Gamma(\gamma)} .
\end{aligned}
$$




\section{Some enumerative results implied by Selberg integral}

We note that lemma 1 directly implies several known results by Selberg integral (4). For examples, the number of SYT of rectangular shape $n^{m}$ is

$$
f^{n^{m}}=\frac{(n m) ! F_{n-1} F_{m-1}}{F_{n+m-1}} \int_{0}^{1} t^{n-1}(1-t)^{m-1} d t=\frac{(n m) ! F_{n} F_{m}}{F_{n+m}} ;
$$

and the number of standard shifted Young tableaux $\delta_{n}=(n, n-1, n-2, \cdots, 1)$ is

$$
\begin{aligned}
f^{\delta_{n}} & =f^{n^{n} \backslash \delta_{n-1}}=\frac{\left(\begin{array}{c}
n+1 \\
2
\end{array}\right) !}{F_{n}} \int_{0<t_{1}<\cdots<t_{n}<1} \cdots \int_{1 \leqslant i<j \leqslant n}\left(t_{j}-t_{i}\right) d t_{1} \cdots d t_{n} \\
& =\frac{\left(\begin{array}{c}
n+1 \\
2
\end{array}\right) !}{F_{n}} \prod_{j=0}^{n-1} \frac{\Gamma^{2}\left(\frac{j}{2}+1\right) \Gamma\left(\frac{j+1}{2}\right)}{\Gamma\left(\frac{n+j+3}{2}\right) \Gamma\left(\frac{1}{2}\right)}=\left(\begin{array}{c}
n+1 \\
2
\end{array}\right) ! \prod_{j=0}^{n-1} \frac{j !}{(2 j+1) !},
\end{aligned}
$$

the last equality is from $\Gamma\left(k+\frac{1}{2}\right)=\frac{(2 k) !}{4^{k} k !} \Gamma\left(\frac{1}{2}\right)$.

\subsection{Panova's and Adin et al.'s results}

Adin et al. [1] and G. Panova [8] derived the product formula of the number of SYT of truncated shape $(n+k)^{m+k} \backslash\left(k^{k-1}, k-1\right)$, while Panova obtained the number of SYT of truncated shape $n^{m} \backslash \delta_{k}$. These two truncated shapes are illustrated as follows

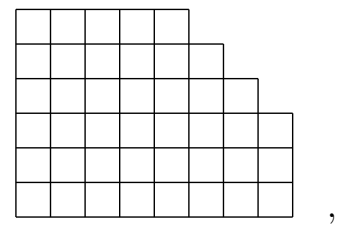

$n^{m} \backslash \delta_{k}$

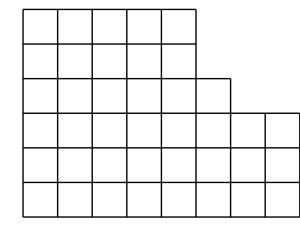

$(n+k)^{m+k} \backslash\left(k^{k-1}, k-1\right)$

Using schur function and computing the volume of a polytope, Panova obtained the following product formula of the number of a rectangle truncated by a staircase.

Proposition 3. [8, Thm.2] The number of standard tableaux of truncated straight shape $n^{m} \backslash \delta_{k}(n \leqslant m)$ is

$$
\left(\begin{array}{c}
m n-\left(\begin{array}{c}
k+1 \\
2
\end{array}\right) \\
m(n-k-1)
\end{array}\right) f^{(n-k-1)^{m}} g_{(m, m-1, \cdots, m-k)} \frac{E_{1}(k+1, m, n-k-1)}{E_{1}(k+1, m, 0)},
$$

where $f^{(n-k-1)^{m}}$ is the number of SYT of rectangle $(n-k-1)^{m}, g_{(m, m-1, \cdots, m-k)}$ is the number of standard tableaux of shifted shape $(m, m-1, \cdots, m-k)$, and

$$
E_{1}(r, p, s)= \begin{cases}\prod_{r<l<2 p-r+2} \frac{1}{(l+2 s)^{r / 2}} \prod_{2 \leqslant l \leqslant r} \frac{1}{((l+2 s)(2 p-l+2+2 s))^{\lfloor l / 2\rfloor}}, & r \text { even } \\ \frac{((r-1) / 2+s) !}{(p-(r-1) / 2+s) !} E_{1}(r-1, p, s), & r \text { odd } .\end{cases}
$$


Combining (1) and (3)-(4), we immediately obtain the following simplified form of Panova's product formula (5).

Theorem 4. The number of SYT of truncated shape $n^{m} \backslash \delta_{k-1}$ is

$$
\frac{\left(n m-\left(\begin{array}{l}
k \\
2
\end{array}\right)\right) ! F_{n-k} F_{m-k}}{F_{n+m-k}} \prod_{j=0}^{k-1} \frac{\Gamma\left(n-k+1+\frac{j}{2}\right) \Gamma\left(m-k+1+\frac{j}{2}\right) \Gamma\left(\frac{j+1}{2}\right)}{\Gamma\left(n+m-2 k+2+\frac{k-1+j}{2}\right) \Gamma\left(\frac{1}{2}\right)} .
$$

Proof. It is clear that

$$
\begin{aligned}
& f^{n^{m} \backslash \delta_{k-1}} \\
& =\frac{\left(n m-\left(\begin{array}{c}
k \\
2
\end{array}\right)\right) ! F_{n-k} F_{m-k}}{F_{n+m-k}} \int_{0<t_{1}<\cdots<t_{k}<1} \ldots \int_{i=1} \prod_{i}^{k} t_{i}^{n-k}\left(1-t_{i}\right)^{m-k} \prod_{1 \leqslant i<j \leqslant k}\left(t_{j}-t_{i}\right) d t_{1} \cdots d t_{k},
\end{aligned}
$$

and the multiple integral in above is Selberg integral $\frac{1}{k !} S_{k}\left(n-k+1, m-k+1, \frac{1}{2}\right)$.

Similarly, there is a simple proof of the number of SYT of rectangle truncated by nearly a square.

Proposition 5. [1, Cor.5.6][8, Thm.3]

The number of SYT of truncated shape $(n+k)^{m+k} \backslash\left(k^{k-1}, k-1\right)$ is

$$
\frac{F_{n} F_{m} F_{k}}{F_{n+m+k}} \cdot \frac{(n m+n k+m k+1) !(n k) !(m k) !}{(n k+m k+1) !} .
$$

Proof. For the rectangle truncated by a square minus a box, we write the variable $t$ corresponding to the reminder box $(k, n+1)$. From (3) we have

$$
f^{(n+k)^{m+k} \backslash\left(k^{k-1}, k-1\right)}=(n m+n k+m k+1) ! \int_{0}^{1} D_{m+k}(t) d t
$$

where

$$
D_{m+k}(t)=\operatorname{det}\left(\begin{array}{ll}
\frac{t^{n-i+j}}{(n-i+j) !}, & 1 \leqslant i \leqslant k \\
\frac{1}{(n+k-i+j) !}, & k+1 \leqslant i \leqslant m+k
\end{array}\right)_{j=1}^{m+k} .
$$

Similar to the proof of (3), it is not difficult to derive

$$
D_{m+k}(t)=\frac{F_{n} F_{m} F_{k}}{F_{n+m+k}} t^{n k}(1-t)^{m k}
$$

by induction. Therefore (7) is implied by Euler's beta integral. 


\subsection{Skew SYT $\left((n+k)^{r+1}, n^{m-1}\right) /(n-1)^{r}$ truncated by a rectangle}

Consider the skew SYT $S=\left((n+k)^{r+1}, n^{m-1}\right) /(n-1)^{r}, n, m \geqslant 2 ; k, r \geqslant 0$. Write the variable $t$ corresponding to the box $(r+1, n)$, illustrated as

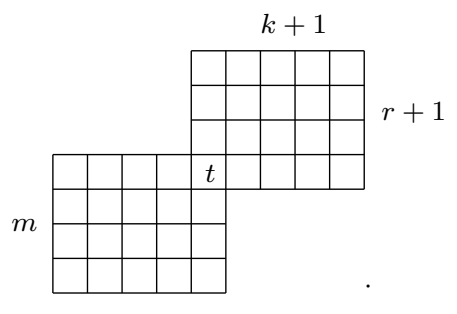

From the hook-length formula or Lemma 1, the number $f^{S}$ is known to be

$$
\frac{F_{n-1} F_{m-1}}{F_{n+m-1}} \cdot \frac{F_{k} F_{r}}{F_{k+r+1}} \cdot \frac{(n m+k+r+k r) !(n+r-1) !(m+k-1) !}{(n+m+k+r-1) !} .
$$

In this section we consider three truncated shapes of $S$, illustrated as

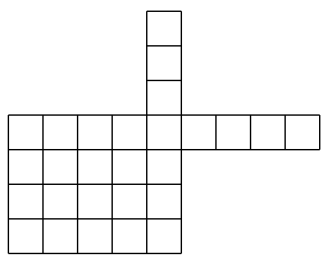

$S \backslash k^{r}$,

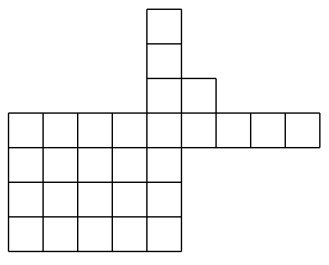

$S \backslash\left(k^{r-1}, k-1\right)$,

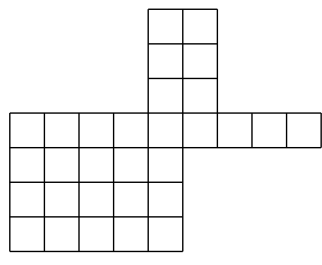

$S \backslash(k-1)^{r}$

Lemma 1 directly implies the following results.

Theorem 6. For $n \geqslant m \geqslant 2 ; k, r \geqslant 0$, the number of skew SYT of truncated shape $\left((n+k)^{r+1}, n^{m-1}\right) /(n-1)^{r} \backslash k^{r}$ is

$$
\frac{F_{n-1} F_{m-1}}{F_{n+m-1}} \cdot \frac{(n m+k+r) !(n+r-1) !(m+k-1) !}{(n+m+k+r-1) ! k ! r !} .
$$

Proof. Write the variable $t$ corresponding to the box $(r+1, n)$, then

$$
f^{S \backslash k^{r}}=\frac{(n m+k+r) ! F_{n-1} F_{m-1}}{F_{n+m-1}} \int_{0}^{1} t^{n-1}(1-t)^{m-1} \frac{t^{r}}{r !} \frac{(1-t)^{k}}{k !} d t,
$$

(9) follows from Euler's beta integral.

Theorem 7. For $n \geqslant m \geqslant 2 ; k, r \geqslant 1$, the number of skew SYT of truncated shape $\left((n+k)^{r+1}, n^{m-1}\right) /(n-1)^{r} \backslash\left(k^{r-1}, k-1\right)$ is

$$
\frac{(n m+k+r+1) ! F_{n} F_{m}}{(n+m+k+r) ! F_{n+m-1}}\left[\left(\begin{array}{c}
n+r-1 \\
r
\end{array}\right)\left(\begin{array}{c}
m+k \\
k+1
\end{array}\right)+\left(\begin{array}{c}
n+r \\
r+1
\end{array}\right)\left(\begin{array}{c}
m+k-1 \\
k
\end{array}\right)\right] .
$$


Proof. Write the variables $x, t, y$ corresponding to the boxes $(r, n),(r+1, n),(r+1, n+1)$ respectively, then

$$
\begin{aligned}
f^{S \backslash\left(k^{r-1}, k-1\right)}= & \frac{(n m+k+r+1) ! F_{n-1} F_{m-1}}{F_{n+m-1}} \times \\
& \iiint_{0<x<t<y<1} t^{n-1}(1-t)^{m-1} \frac{x^{r-1}}{(r-1) !} \frac{(1-y)^{k-1}}{(k-1) !}(y-x) d x d y d t,
\end{aligned}
$$

and the triple integral in above is easy to evaluate by Euler's beta integral.

Theorem 8. For $n \geqslant m \geqslant 2, k \geqslant 1, r \geqslant 0$, the number of skew SYT of truncated shape $\left((n+k)^{r+1}, n^{m-1}\right) /(n-1)^{r} \backslash(k-1)^{r}$ is

$$
\frac{(n m+k+2 r) ! F_{n} F_{m}}{(n+m+k+2 r-1) ! F_{n+m-1}} \times C_{r},
$$

where

$$
C_{r}=\sum_{i=0}^{r} \frac{r+1-i}{r+1}\left(\begin{array}{c}
r+i \\
i
\end{array}\right)\left(\begin{array}{c}
n+r-1+i \\
r+i
\end{array}\right)\left(\begin{array}{c}
m+k+r-1-i \\
k+r-i
\end{array}\right) .
$$

Proof. Similar to Theorem 7, write the variables $x, t, y$ corresponding to the boxes $(r, n)$, $(r+1, n),(r+1, n+1)$ respectively, then

$$
\begin{aligned}
f^{S \backslash(k-1)^{r}=} & \frac{(n m+k+2 r) ! F_{n-1} F_{m-1}}{F_{n+m-1}} \times \\
& \iiint_{0<x<t<y<1} t^{n-1}(1-t)^{m-1} \frac{(1-y)^{k-1}}{(k-1) !}\left[\frac{x^{r-1} y^{r}}{(r-1) ! r !}-\frac{x^{r} y^{r-1}}{r !(r-1) !}\right] d x d y d t .
\end{aligned}
$$

Therefore, (11) follows from the well-known incomplete beta integral:

$$
\int_{t}^{1} \frac{y^{r}}{r !} \frac{(1-y)^{k-1}}{(k-1) !} d y=\sum_{i=0}^{r} \frac{t^{i}}{i !} \frac{(1-t)^{k+r-i}}{(k+r-i) !}, 0<t<1 .
$$

\section{New enumerative results of SYT of truncated shapes}

In this section we use Lemma 1 and combinatorial summation to derive the number of SYT of truncated shapes. Consider the following two shapes:

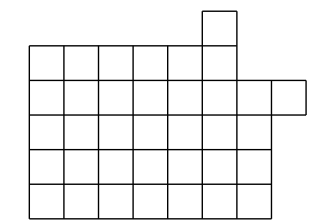

$\left((n+1)^{3}, n^{m-2}\right) /(n-2) \backslash\left(2^{2}\right)$,

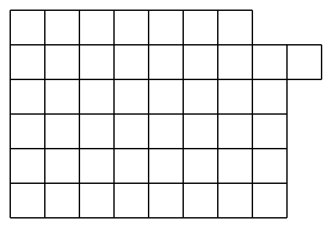

$\left((n+1)^{2}, n^{m-2}\right) \backslash(2)$. 
Theorem 9. For $n \geqslant m \geqslant 2$, the number of skew SYT of truncated shape $((n+$ $\left.1)^{3}, n^{m-2}\right) /(n-2) \backslash\left(2^{2}\right)$ is

$$
\frac{(n m+1) ! F_{n} F_{m}}{2 F_{n+m}}\left[\frac{(2 m-1)\left(\begin{array}{c}
n+m-2 \\
n-1
\end{array}\right)^{2}}{\left(\begin{array}{c}
2 n+2 m-3 \\
2 n-1
\end{array}\right)}-1\right] .
$$

Theorem 10. For $n \geqslant m \geqslant 2$, the number of $S Y T$ of truncated shape $\left((n+1)^{2}, n^{m-2}\right) \backslash$ is

$$
\frac{(n m) ! F_{n} F_{m}}{2 F_{n+m}}\left[\frac{(2 m-1)\left(\begin{array}{c}
n+m-2 \\
n-1
\end{array}\right)^{2}}{\left(\begin{array}{c}
2 n+2 m-4 \\
2 n-2
\end{array}\right)}-1\right] .
$$

\subsection{Combinatorial identities}

First, we prove the following combinatorial identity.

Lemma 11. For any $1 \leqslant M \leqslant N$,

$$
\sum_{i=0}^{M-1}\left(\begin{array}{c}
M-1+i \\
i
\end{array}\right)\left(\begin{array}{c}
2 N-M-i \\
N-i
\end{array}\right)=\frac{1}{2}\left(\begin{array}{c}
2 N \\
N
\end{array}\right)=\left(\begin{array}{c}
2 N-1 \\
N
\end{array}\right)
$$

Proof. From the classical identity [7, (3.2)]

$$
\sum_{k=0}^{n}\left(\begin{array}{c}
x+k \\
k
\end{array}\right)\left(\begin{array}{c}
y+n-k \\
n-k
\end{array}\right)=\left(\begin{array}{c}
x+y+n+1 \\
n
\end{array}\right)
$$

we have

$$
\left(\begin{array}{c}
2 N \\
N
\end{array}\right)=\sum_{i=0}^{M-1}\left(\begin{array}{c}
M-1+i \\
i
\end{array}\right)\left(\begin{array}{c}
2 N-M-i \\
N-i
\end{array}\right)+\sum_{i=M}^{N}\left(\begin{array}{c}
M-1+i \\
i
\end{array}\right)\left(\begin{array}{c}
2 N-M-i \\
N-i
\end{array}\right) .
$$

It is clear that Catalan identity [7, (3.165)]:

$$
\sum_{k=0}^{n}\left(\begin{array}{c}
p-k \\
p-n
\end{array}\right)\left(\begin{array}{c}
q+k+1 \\
m
\end{array}\right)=\sum_{k=0}^{m}\left(\begin{array}{c}
q-k \\
q-m
\end{array}\right)\left(\begin{array}{c}
p+k+1 \\
n
\end{array}\right)
$$

implies

$$
\begin{aligned}
& \sum_{i=M}^{N}\left(\begin{array}{c}
M-1+i \\
i
\end{array}\right)\left(\begin{array}{c}
2 N-M-i \\
N-i
\end{array}\right)=\sum_{k=0}^{N-M}\left(\begin{array}{c}
2 M-2+k+1 \\
M-1
\end{array}\right)\left(\begin{array}{c}
2 N-2 M-k \\
(2 N-2 M)-(N-M)
\end{array}\right) \\
& =\sum_{k=0}^{M-1}\left(\begin{array}{c}
2 M-2-k \\
M-1-k
\end{array}\right)\left(\begin{array}{c}
2 N-2 M+k+1 \\
N-M
\end{array}\right)=\sum_{i=0}^{M-1}\left(\begin{array}{c}
M-1+i \\
i
\end{array}\right)\left(\begin{array}{c}
2 N-M-i \\
N-i
\end{array}\right),
\end{aligned}
$$

the proof of Lemma 11 is complete. 
Corollary 12. (1). For any $1 \leqslant M \leqslant N-1$,

$$
\begin{aligned}
\sum_{i=0}^{M-1}\left(\begin{array}{c}
M-1+i \\
i
\end{array}\right) & \left(\begin{array}{c}
2 N-M-1-i \\
N-i
\end{array}\right) \\
= & \frac{1}{2}\left(\begin{array}{c}
2 N-1 \\
N
\end{array}\right)-\frac{M}{N}\left(\begin{array}{c}
2 M-1 \\
M
\end{array}\right)\left(\begin{array}{c}
2 N-2 M-1 \\
N-M
\end{array}\right) .
\end{aligned}
$$

(2). For any $1 \leqslant M \leqslant N-2$,

$$
\begin{aligned}
\sum_{i=0}^{M-1}\left(\begin{array}{c}
M-1+i \\
i
\end{array}\right) & \left(\begin{array}{c}
2 N-M-2-i \\
N-i
\end{array}\right) \\
= & \frac{1}{2}\left(\begin{array}{c}
2 N-2 \\
N
\end{array}\right)-\frac{M}{N}\left(\begin{array}{c}
2 M-1 \\
M
\end{array}\right)\left(\begin{array}{c}
2 N-2 M-1 \\
N-M
\end{array}\right) .
\end{aligned}
$$

Proof. Notice that the left side of (15) is equal to

$$
\frac{N-M}{2 N} \sum_{i=0}^{M-1}\left(\begin{array}{c}
M-1+i \\
i
\end{array}\right)\left(\begin{array}{c}
2 N-M-i \\
N-i
\end{array}\right)+\frac{M}{2 N} \sum_{i=0}^{M-1}\left(\begin{array}{c}
M+i \\
i
\end{array}\right)\left(\begin{array}{c}
2 N-M-1-i \\
N-i
\end{array}\right) \text {, }
$$

then, the equality (15) is implied by (14).

The equality (16) follows from

$$
\left(\begin{array}{c}
2 N-M-2-i \\
N-i
\end{array}\right)=\left(\begin{array}{c}
2 N-M-1-i \\
N-i
\end{array}\right)-\left(\begin{array}{c}
2 N-M-2-i \\
N-1-i
\end{array}\right) .
$$

\subsection{Proofs of Theorem 9-10}

Proof of Theorem 9. Write the variable $x$ corresponding to the box $(2, n-1)$, and $y$ corresponding to $(3, n)$ respectively. From Lemma 1, we know that $f^{\left((n+1)^{3}, n^{m-2}\right) /(n-2) \backslash\left(2^{2}\right)}$ is equal to

$$
\frac{(n m+1) ! F_{n-2} F_{m-2}}{F_{n+m-2}} \iint_{0<x<y<1} x^{n-1}(1-x)^{m-2} y^{n-2}(1-y)^{m-1}(y-x) d x d y .
$$

Noticing that incomplete beta integral

$$
\int_{x}^{1} \frac{y^{r-1}}{(r-1) !} \frac{(1-y)^{n-r}}{(n-r) !} d y=\sum_{k=0}^{r-1} \frac{x^{k}}{k !} \frac{(1-x)^{n-k}}{(n-k) !}, 0<x<1
$$


implies

$$
\begin{aligned}
& \iint_{0<x<y<1} x^{a}(1-x)^{n-a} y^{b}(1-y)^{m-b} d x d y \\
& =\frac{a !(n-a) ! b !(m-b) !}{(n+m+2) !} \sum_{i=0}^{b}\left(\begin{array}{c}
a+i \\
i
\end{array}\right)\left(\begin{array}{c}
n+m+1-a-i \\
m+1-i
\end{array}\right)
\end{aligned}
$$

which follows that

$$
f^{\left((n+1)^{3}, n^{m-2}\right) /(n-2) \backslash\left(2^{2}\right)}=\frac{(n m+1) ! F_{n} F_{m}}{(2 n+2 m-3) ! F_{n+m-2}} \times\left[(n-1) A_{1}-n A_{2}\right]
$$

where

$$
A_{1}=\sum_{i=0}^{n-1}\left(\begin{array}{c}
n-1+i \\
i
\end{array}\right)\left(\begin{array}{c}
n+2 m-3-i \\
n+m-1-i
\end{array}\right), \quad A_{2}=\sum_{i=0}^{n-2}\left(\begin{array}{c}
n+i \\
i
\end{array}\right)\left(\begin{array}{c}
n+2 m-4-i \\
n+m-2-i
\end{array}\right) .
$$

Taking $M=n, N=n+m-1$ in (15), then

$$
A_{1}=\frac{1}{2}\left(\begin{array}{c}
2 n+2 m-3 \\
n+m-1
\end{array}\right)-\frac{n}{n+m-1}\left(\begin{array}{c}
2 n-1 \\
n
\end{array}\right)\left(\begin{array}{c}
2 m-3 \\
m-1
\end{array}\right) .
$$

Similarly, (14) and (15) imply that

$$
\begin{aligned}
& A_{2}+\left(\begin{array}{c}
2 n-1 \\
n-1
\end{array}\right)\left(\begin{array}{c}
2 m-3 \\
m-1
\end{array}\right)+\left(\begin{array}{c}
2 n \\
n
\end{array}\right)\left(\begin{array}{c}
2 m-4 \\
m-2
\end{array}\right) \\
& =\sum_{i=0}^{n}\left(\begin{array}{c}
n+i \\
i
\end{array}\right)\left(\begin{array}{c}
n+2 m-3-i \\
n+m-1-i
\end{array}\right)-\sum_{i=0}^{n}\left(\begin{array}{c}
n+i \\
i
\end{array}\right)\left(\begin{array}{c}
n+2 m-4-i \\
n+m-1-i
\end{array}\right) \\
& =\frac{1}{2}\left(\begin{array}{c}
2 n+2 m-3 \\
n+m-1
\end{array}\right)+\frac{n+1}{n+m-1}\left(\begin{array}{c}
2 n+1 \\
n+1
\end{array}\right)\left(\begin{array}{c}
2 m-5 \\
m-2
\end{array}\right),
\end{aligned}
$$

therefore,

$$
(n-1) A_{1}-n A_{2}=\frac{n m}{2(n+m-1)}\left(\begin{array}{c}
2 n-1 \\
n
\end{array}\right)\left(\begin{array}{c}
2 m-1 \\
m
\end{array}\right)-\frac{1}{2}\left(\begin{array}{c}
2 n+2 m-3 \\
n+m-1
\end{array}\right) .
$$

We complete the proof of (12).

Proof of Theorem 10. A similar argument to that above shows that

$$
f^{\left((n+1)^{2}, n^{m-2}\right) \backslash(2)}=\frac{(n m) ! F_{n} F_{m}}{(2 n+2 m-4) ! F_{n+m-2}} \times\left[A_{1}-A_{2}\right]
$$

where

$$
A_{1}=\sum_{i=0}^{n-1}\left(\begin{array}{c}
n-2+i \\
i
\end{array}\right)\left(\begin{array}{c}
n+2 m-3-i \\
n+m-1-i
\end{array}\right), \quad A_{2}=\sum_{i=0}^{n-2}\left(\begin{array}{c}
n-1+i \\
i
\end{array}\right)\left(\begin{array}{c}
n+2 m-4-i \\
n+m-2-i
\end{array}\right) .
$$


Taking $M=n, N=n+m-2$ in (14), then

$$
A_{2}=\frac{1}{2}\left(\begin{array}{c}
2 n+2 m-4 \\
n+m-2
\end{array}\right)-\left(\begin{array}{c}
2 n-2 \\
n-1
\end{array}\right)\left(\begin{array}{c}
2 m-3 \\
m-1
\end{array}\right) .
$$

On the other hand, Taking $M=n-1$ and $N=n+m-1$ in (16) implies

$$
A_{1}=\frac{1}{2}\left(\begin{array}{c}
2 n+2 m-4 \\
n+m-1
\end{array}\right)+\frac{m-n}{2(n+m-1)}\left(\begin{array}{c}
2 n-2 \\
n-1
\end{array}\right)\left(\begin{array}{c}
2 m-2 \\
m-1
\end{array}\right) .
$$

Therefore,

$$
A_{1}-A_{2}=\frac{2 m-1}{2(n+m-1)}\left(\begin{array}{c}
2 n-2 \\
n-1
\end{array}\right)\left(\begin{array}{c}
2 m-2 \\
m-1
\end{array}\right)-\frac{1}{2(n+m-1)}\left(\begin{array}{c}
2 n+2 m-4 \\
n+m-2
\end{array}\right) .
$$

The proof of (13) is complete.

\section{Acknowledgements}

The author expresses his sincere thanks to the anonymous referee for the helpful comments and useful suggestions which led to an improvement on an earlier version of this manuscript.

\section{References}

[1] Ron M. Adin, Ronald C. King, and Yuval Roichman. Enumeration of standard Young tableaux of certain truncated shapes. Electron. J. Combin., 18(2) (2011), \# P20.

[2] Y. Baryshnikov and D. Romik. Enumeration formulas for Young tableaux in a diagonal strip. Israel J. Math., 178:157-186, 2010.

[3] H. A. David, H. N. Nagaraja. Order Statistics. 3rd ed. John Wiley \& Sons, Inc., Hoboken, New Jersey, 2003.

[4] N. Elkies. On the sums $\sum_{k=-\infty}^{\infty}(4 k+1)^{-n}$. Amer. Math. Monthly., 110:561-573, 2003.

[5] J. S. Frame, G. de B. Robinson, and R. M. Thrall. The hook graphs of the symmetric group. Canad. J. Math., 6:317-324, 1954.

[6] Peter J. Forrester and S. Ole Warnaar. The importance of the Selberg integral. Bull. Amer. Math. Soc. (N.S.), 45:489-534, 2008.

[7] H. W. Gould. Combinatorial Identities. Morgantown, W. Va. 1972.

[8] Greta Panova. Tableaux and plane partitions of truncated shapes. Adv. Appl. Math., 49:196-217, 2012.

[9] Amitai. Regev. Asymptotic values for degrees associated with strips of Young diagrams. Adv. Math. 41:115-136, 1981. 
[10] A. Selberg. Bemerkninger om et multipelt integral. Norsk. Mat. Tidsskr., 24:71-78, 1944.

[11] Richard P. Stanley. Two poset polytopes. Discrete Comput. Geom., 1:9-23, 1986.

[12] Richard P. Stanley, Enumerative Combinatorics. vol. 2, Cambridge University Press, New York, 1999. 